\title{
CORRESPONDENCE
}

Letters of 600 words or less, with minimal allowance for tables, figures, and references, will be considered for publication. Rules regarding prior publication, conflict of interest, etc., are the same as for full manuscripts. All letters are subject to editing and condensation. Proofs will not be distributed.

\section{Dosage, Threshold, and Duration of ECT}

To the Editor:

The article by Sackeim et al. (1987) entitled "Studies of Dosage. Seizure Threshold, and Seizure Duration in ECT" is one of the most stimulating articles on ECT I have read in recent times. Nevertheless, the research methodology used in the paper and the conclusions proposed by the authors need clarification. viz:

1. The inclusion criteria for the study was a 24-item Hamilton Depression Rating of 18 or more points, which is a relatively low HRSD score for an ECT cohort. The authors state that there were no differences in the Hamilton Depression scores between the unilateral and bilateral treatment groups, but present only the overall mean (HRSD 31.09 \pm 7.76 ). It would be of interest to know the actual mean data per group and whether baseline difference could explain some of the outcome variability noted in the unilateral group.

2. How did the authors define treatment response? Was it done on a clinical basis or did they use Hamilton Depression criteria? Definitions for treatment response were not given in the description of the methods.

3. The authors allowed the use of prn lorazepam up to $1 \mathrm{mg} \mathrm{b.i.d.} \mathrm{Like} \mathrm{other} \mathrm{benzodiazepines,} \mathrm{it} \mathrm{is}$ well known for its antiseizure effects. The use of benzodiazepines during ECT has been associated with failure to induce seizures and shorter seizure duration, both of which contribute to poor treatment outcome with ECT (d'Elia et al. 1983). Lorazepam has a half-life between 12 and $20 \mathrm{hr}$, and thus shows a lesser tendency to accumulate in patients; in aged patients, who are not infrequently referred for ECT, accumulation could occur (Shader and Greenblat, 1981). Was the intake of lorazepam, number of doses, and period of use different between the unilateral and bilateral groups? Did the authors correlate seizure threshold and seizure duration with lorazepam use? Could the authors reanalyze their main conclusions in a group of patients free from lorazepam? The inhibitory effects of benzodiazepines might be exaggerated by the method of titration used by the authors.

4. The authors did not discuss the indications for referring these patients for $\mathrm{ECT}$. Were these patients medication nonresponders or was ECT chosen as the initial treatment modality? Even though it is not customary to establish this difference, in our experience the response to ECT is greater in those patients in whom ECT is the initial treatment modality. Were both groups, unilateral and bilateral, comparable with regard to the number of previous medication nonresponder patients?

5. Did the authors compare cognitive functions preand post-ECT? Were unilateral-bilateral differences found?

The dramatically greater clinical efficacy of bilateral ECT described by the authors is puzzling. Many elinicians preseribe unilateral $\mathrm{ECT}$ under the assumption that it is as effective as bilateral ECT, even though the unilateral course may consist of more treatments. Bilateral electrode placement has been associated with more complaints of post-ECT $\operatorname{cog}$ nitive abnormalities, which may persist for some months after the course of ECT. The conclusions of the paper by Sackeim et al. may have a great impact on these assumptions. Therefore, methodological purity is of grear relevance.

Department of Psychiatry

University of Michigan

Ann Arbor, Michigan 48109

Leon $J$. Grunhaus

\section{References}

d'Elia G, Ottosson JO, Stromgren L (1983): Present practice of electroconvulsive therapy in Scandinavia. Arch Gen Psychiatry 40:577-581. 
Sackeim HA, Decina P, Portnoy S, Neeley P, Malitz S (1987): Studies of dosage, seizure threshold, and seizure duration in ECT. Biol Psychiatry 22:249-268.
Shader RI, Greenblat DJ (1981): The use of benzodiazepines in clinical practice. Br J Clin Pharmacol 11:5S-9S.
To the Editor:

Sackeim and colleagues are to be congratulated for the wealth of information reported in their recent paper (1987). Using a MECTA instrument, they demonstrated clinically that the units of charge are a better indicator of the electrostimulatory dose than the ergometric units, watt seconds or joules commonly used to indicate the dose in electroconvulsive therapy (ECT). Strictly speaking, this is incorrect. They did not specify the type of MECTA instrument, but they specified that bidirectional pulse current was used. In alternating current, the net charge is close to zero regardless of the current duration, as each pulse of halfwave negates the charge of the previous one. To avoid this problem, one has to use a function of the absolute value of the charge (which Sackeim et al. seem to have done), or to square the current, as the stimulus energy increases with the square of the current. Gordon (1981) discussed the problems inherent in both methods of ECT dosimetry and suggested the recording of all parameters (voltage, current, joules, coulombs). In our experiments (Hyrman et al. 1985), we have also used units of charge, millicoulombs, to represent the dose with unidirectional pulses. This was quite adequate as long as the current remained constant. However, for comparison of various current patterns in ECT, we proposed a standard electrostimulatory dose unit (ESD) that would be arrived at using the formula:

$$
\mathrm{ESD}=P^{2} d t(k)
$$

where $l$ denotes the current in amps and $d t$ the differential time. The constant $k=1000$ is used in order to arrive at units of convenient size. The average treatment dose with sine wave instrumentation would be about $500 \mathrm{ESD}$ units, and a brief pulse machine, such as MECTA, would require a dose of about 200 units. The Ultra Low Energy ECT instrument (ULEECTA), promoted by this writer, is calibrated in ESD units. In an 18-month clinical trial, the average dose is in the neighborhood of 50 ESD units.

More research is nceded to clarify the effects of the ECT stimulus; adopting a standard dosimetric unit for ECT would facilitate both the research and the communication of research findings.

V. Hyrman

Department of Psychiatry

Royal Columbian Hospital

260 Sherbrooke Street

New Westminster, B.C.

V3L 3W7 Canada

\section{References}

Hyrman V, Palmer LH, Cernik J, Jetelina J (1985): ECT: The search for the perfect stimulus. Biol Psychiatry 20:634-645.

Sackcim HA, Decina P, Portnoy S, Neclcy P, Malitz S (1987): Studies of dosage, seizure threshold, and seizure duration in ECT. Biol Psychiatry 22:249-268.

\section{Response}

To the Editor:

I thank Drs. Grunhaus and Hyrman for their kind remarks about our paper, "Studies of Dosage, Seizure Threshold, and Seizure Duration in ECT" (Sackeim et al. 1987a). Space limitations prevent detailed response to each of the requests for additional information. In any case, most of the requested details have been provided in prior publications and in papers that are in press (Malitz et al. 1986; Sackeim et al. 1986a, 1986b, 1987b, 1987c, 1987d; Steif et al. 1986). The queries raised by Dr. Grunhaus will be responded to first.

1. The sample was mostly composed of patients with severe, recurrent depressive cpisodes. Of 52 patients, $42(81 \%)$ had pretreatment HRSD scores of 25 or greater. The mean pretreatment score was 31.54 (SD 8.37) in the unilateral right ECT group and 30.68 (SD 6.97) in the bilateral ECT group, a nonsignificant difference. Pretreatment scores were not associated with posttreatment scores, $r(50)$ $=0.05$, NS (Malitz et al. 1986; Sackeim et al. 